\title{
CORRECTION
}

\section{Correction to: Early Prediction of Malignant Edema After Successful Recanalization in Patients with Acute Ischemic Stroke}

Changyi Wang ${ }^{1,2}$, Qiange Zhu ${ }^{3}$, Ting Cui ${ }^{1}$, Lu Wang ${ }^{2}$, Tang Yang ${ }^{1}$, Zilong Hao ${ }^{1}$, Simiao Wu ${ }^{1}$, Hongbo Zheng ${ }^{1}$, Fayun $\mathrm{Hu}^{1 *}$ and $\mathrm{Bo} \mathrm{Wu}^{1 *}$ (D)

๑ 2022 Springer Science+Business Media, LLC, part of Springer Nature and Neurocritical Care Society

\section{Correction to: Neurocrit Care https://doi.org/10.1007/s12028-021-01380-4}

The authors would like to correct errors in Table 1. Regarding the components of PCHDs, we should have only included patients with PCHDs. However, patients who did not have PCHDs were included in the original version. The correct data should be as follows. 
Table 1 Patient characteristics stratified by the presence of malignant brain edema

\begin{tabular}{|c|c|c|c|c|}
\hline & Overall & No malignant brain edema & Malignant brain edema & $p$ value \\
\hline Component of PCHDs, n (\%)* & & & & $<0.001$ \\
\hline Contrast staining & $107(43.3)$ & $98(52.7)$ & $9(14.8)$ & \\
\hline Hemorrhage & $118(47.8)$ & $85(45.7)$ & $33(54.1)$ & $<0.001$ \\
\hline $\mathrm{HI}-1$ & $15(12.7)$ & $15(17.6)$ & $0(0)$ & \\
\hline $\mathrm{H}-2$ & $32(27.1)$ & $32(37.6)$ & $0(0)$ & \\
\hline $\mathrm{PH}-1$ & $51(43.2)$ & $32(37.6)$ & $19(57.6)$ & \\
\hline $\mathrm{PH}-2$ & $20(17.0)$ & $6(7.2)$ & $14(42.4)$ & \\
\hline Undetermined & $22(8.9)$ & $3(1.6)$ & $19(31.1)$ & \\
\hline
\end{tabular}

*Only patents with PCHD were included $(n=247)$

$\mathrm{PCHD}$, postinterventional cerebral hyperdensity; $\mathrm{HI}$, hemorrhagic infarct; and $\mathrm{PH}$, parenchymal hematoma

\section{Author details}

${ }^{1}$ Department of Neurology, Center of Cerebrovascular Diseases, West China Hospital, Sichuan University, Chengdu, Sichuan, China. ${ }^{2}$ Departement of Rehabilitation Medicine Center, West China Hospital, Sichuan University,
Chengdu, Sichuan, China. ${ }^{3}$ The Second Department of Neurology, Shaanxi Provincial People's Hospital, Xi'an, Shaanxi, China.

\section{Publisher's Note}

Springer Nature remains neutral with regard to jurisdictional claims in published maps and institutional affiliations.

Published: 11 January 2022 